\title{
Thermal transistor: Heat flux switching and modulating
}

\author{
Wei Chung $\mathrm{LO}^{1,4}$, Lei WANG ${ }^{1,2}$ and Baowen $\mathrm{LI}^{1,3}$ \\ ${ }^{1}$ Department of Physics and Centre for Computational Science and Engineering, \\ National University of Singapore, Singapore 117542, Republic of Singapore \\ ${ }^{2}$ Department of Physics, Renmin University of China, Beijing 100872, P. R. China \\ ${ }^{3}$ NUS Graduate School for Integrative Science and Engineering, Singapore 117597, Republic of Singapore \\ ${ }^{4}$ Institute of High Performance Computing, 1 Science Park Road, Singapore 117528
}

\begin{abstract}
Thermal transistor is an efficient heat control device which can act as a heat switch as well as a heat modulator. In this paper, we study systematically one-dimensional and two-dimensional thermal transistors. In particular, we show how to improve significantly the efficiency of the onedimensional thermal transistor. The study is also extended to the design of two-dimensional thermal transistor by coupling different anharmonic lattices such as the Frenkel-Kontorova and the FermiPasta-Ulam lattices. Analogy between anharmonic lattices and single-walled carbon nanotube is drawn and possible experimental realization with multi-walled nanotube is suggested.
\end{abstract}

PACS numbers: 85.90.+h,07.20.Pe, 07.20.-n,63.22.+m

\section{INTRODUCTION}

Much attention has been devoted in the past years to the study of heat conduction in low-dimensional systems [1]. Such study is not only important for understanding the fundamentals of statistical mechanics, but also for potential applications in heat control and management in nanoscale devices.

Indeed, various models for thermal rectifiers/diodes that allow heat to flow easily in one direction have been proposed [2, 3, 4, 5, 6, 7, 8, 9]. Moreover, based on the phenomenon of the negative differential thermal resistance $(N D T R)$ observed in nonlinear lattices, a theoretical model for "thermal transistor" is also proposed [10], which allows us to control heat current (due to phonons) by adjusting the temperature of the gate terminal (or called "gate temperature"). This is similar to the electronic transistor which controls the electric current by adjusting the gate voltage. We should point out that our "thermal transistor" is different from the recent "heat transistor", which controls the heat flux (caused by electrons) via adjusting the gate voltage 11]. Most recently, based on the thermal transistor, the different thermal logic gats have been also realized theoretically [12], which means that the phonons, the heat carrier, can also be used to carry information and processed accordingly.

The purpose of the current paper are two folds. First, we shall discuss how to improve significantly the switching efficiency of 1D thermal transistor; Second, we shall extend the study from $1 \mathrm{D}$ model to $2 \mathrm{D}$ which is closer to laboratory fabricated materials.

\section{ONE-DIMENSIONAL MODEL}

The 1D thermal transistor model consists of three segments $S, D$, and $G$ and each segment is a FrenkelKontorova (FK) lattice [13, 14]. The model configuration, which is shown in Fig. 1(a), is similar to the one proposed in Ref.[10] except for an additional interface coupling $k_{\text {int } S}$. In each segment, we regard the particle that is coupled to heat bath as the first particle and the interface particle as the last particle. The total Hamiltonian of the $1 \mathrm{D}$ model writes

$$
H=H_{S}+H_{D}+H_{G}+H_{\text {int }},
$$

and the Hamiltonian of each segment can be written as $H_{W}=\sum_{i=1}^{N_{W}} \frac{p_{W, i}^{2}}{2 m}+\frac{k_{W}}{2}\left(x_{W, i}-x_{W, i-1}\right)^{2}-\frac{V_{W}}{(2 \pi)^{2}} \cos 2 \pi x_{W, i}$, with $x_{W, i}$ and $p_{W, i}$ denote the displacement from equilibrium position and the conjugate momentum of the $i^{t h}$ particle in segment $W$, where $W$ stands for $S, D$ or $G$. The parameters $k$ and $V$ are the harmonic spring constant and the on-site potential of the FK lattice. We couple the last particle of segment $S, D$, and $G$ to particle $O$ via harmonic springs. Thus $H_{\text {int }}=\frac{p_{O}^{2}}{2 m}-\frac{V_{O}}{(2 \pi)^{2}} \cos 2 \pi x_{O}+$ $\frac{k_{i n t S}}{2}\left(x_{N_{S}}-x_{O}\right)^{2}+\frac{k_{i n t D}}{2}\left(x_{N_{D}}-x_{O}\right)^{2}+\frac{k_{i n t G}}{2}\left(x_{N_{G}}-x_{O}\right)^{2}$. Fixed boundaries are used, i.e., $x_{W, 0}=0$ and each segment consists of 10 particles.

In our simulations, we use the Nosé-Hoover heat baths [15] and we set the mass of each particle and the Boltzmann constant to unity unless otherwise stated. In the nonequilibrium stationary state, the heat flux, which is the rate of energy transport, is constant along each segment and the local equilibrium temperature is given by the time average of the square of particle's velocity per degree of freedom.

We show in Fig. 1 that the model described by Hamiltonian (1) can display different heat controlling features depends on the applied gate temperature, $T_{G}$. In particular, the model can act as a heat switch, namely, the system can be switched from a heat insulator to a heat conductor by just adjusting the gate temperature. As demonstrated in Fig. 1(b), when $T_{G}$ is at 0.01, 0.08, and $0.14, J_{S}=J_{D}$, thus $J_{G}=0$, namely, the control terminal does not provide any current to the system, the system, however is switched from an insulating (off) state, to a semi-conducting (semi-on) state, and conducting (on) state. At these three points, $J_{D, S}$ are $3.8 \times 10^{-7}$, $1.8 \times 10^{-4}$, and $3.2 \times 10^{-4}$, respectively. The ratio of $J_{D, S}$ 

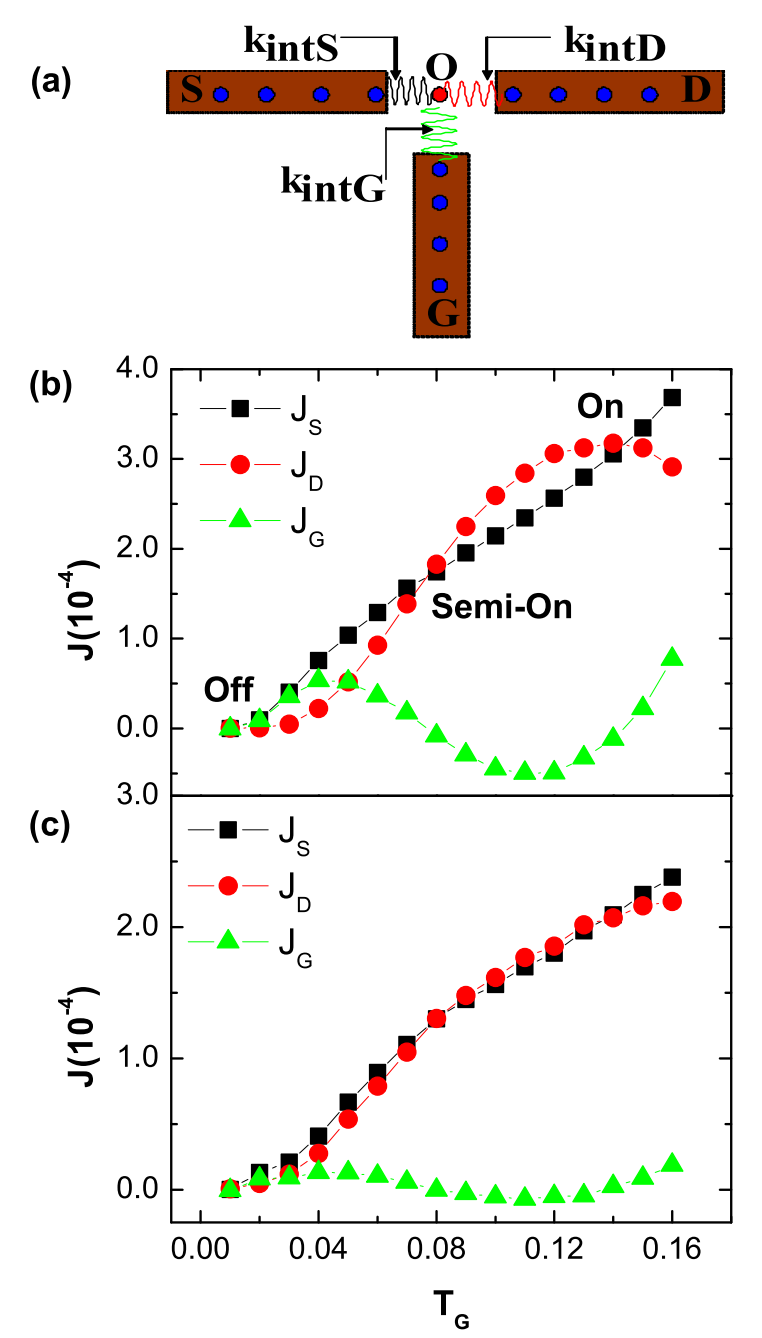

FIG. 1: (a) Configuration of the thermal transistor. Parameters of the system are (b) Thermal switch: $T_{S}=0.01$, $V_{S}=5.00, k_{S}=0.35, k_{\text {int } S}=0.30 ; T_{D}=0.20, V_{D}=1.00, k_{D}=0.20$, $k_{\text {int } D}=0.05 ; V_{G}=5.00, k_{G}=1.00, k_{\text {int } G}=0.20$, and $V_{O}=5.00$. (c) Thermal modulator: all parameters remain the same as in (b) except with $k_{\text {int } G}=0.05$.

at the "on" state to that at the "off" state is considered as the switching efficiency which is about 800 .

We further demonstrate that the model can act as a heat modulator as well in which $J_{D, S}$ can be continuously modulated by the gate temperature. To show this, we recall that for any two weakly coupled lattices, the heat current depends among others, on the strength of the interface coupling used $[3,16]$. Thus, to have $J_{D} \approx J_{S}$, we can reduce the strength of $k_{i n t G}$ so that the heat flow through the control terminal decreases. Apparently, $k_{i n t G}$ should not be too small otherwise $T_{O}$ is not wellcontrolled by $T_{G}$. The numerical result is shown in Fig. $1(\mathrm{c})$.
In our next configuration, we replace segment $D$ with a FPU- $\beta$ lattice whose phonon spectrum is contributed by low frequency vibrations (acoustic phonons) only. The Hamiltonian is given by $H=\sum_{i=1}^{N} \frac{p_{i}^{2}}{2 m}+\frac{k_{F P U}}{2}\left(x_{i}-x_{i-1}\right)^{2}+$ $\frac{\beta}{4}\left(x_{i}-x_{i-1}\right)^{4}$, with $\beta$ being the anharmonicity. We show in Fig. 2(a) that such a setup reduces the heat current at "off" state greatly but affects the heat current at "on" state only slightly with the switching of about 1300 , where $J_{D, S}$ equals to $2.15 \times 10^{-7}$ and $2.82 \times 10^{-4}$ at $T_{G}=0.01$ and 0.14 , respectively. It can be noticed that for this and the former lattice choice in the present model, $J_{D, S}$ at the "off" and "on" state is about $10^{-7}$ and $10^{-4}$, respectively, with a switching of about $10^{3}$, this is a significant improvement compared with previous one [10]. The high switching is mainly a result of the significant reduction of $J_{D, S}$ at the "off" state, which is basically due to the lower $T_{O}$ at "off" state ( in the previous work it is designed at 0.04). Moreover, we found that using lattice with lower heat resistance as terminal $D$ is ideal for achieving a large $J_{D}$. In Fig. 2(b), we show the heat modulating effect with the same setup. Since the demonstrated features of the transistor rely very much on an efficient control of the interface temperature, it would be interesting also to study how the property of the control terminal can affect its functions. In Fig. 3, we show that when we use the FPU- $\beta$ lattice as the control segment, due to the large heat resistance in the segment, temperature $T_{O}$ does not well follow $T_{G}$ thus heat currents in segment $S$ and $D$ do not sensitively depend on $T_{G}$ in the low temperature regime. Only when $T_{G}>0.08$, significant increment of currents and continuous modulation of $J_{D, S}$ are observed. In other words, $J_{D, S}$ can be even more precisely adjusted.

The results presented so far have shown that by choosing the lattice in different segment appropriately, one can improve the switching and modulating efficiency significantly. This message is very important and useful for optimization and experimental realization of the thermal transistor. In the following, we shall extend the study of the thermal transistor to 2D. Such extension is important in application, as it takes into account the interaction between longitudinal and transverse motions and therefore is closer to real material such as thin film etc. However, it is not our intention here to look for optimum switching/ modulating condition for this model.

\section{TWO-DIMENSIONAL MODEL}

The displacement from equilibrium position and the momentum of a particle in 2D lattice are labeled as $\vec{q} \equiv\left\{q^{x}, q^{y}\right\}$ and $\vec{p} \equiv\left\{p^{x}, p^{y}\right\}$, respectively. The relative separation between two particles is $\Delta r=|\vec{r}|=\left|\overrightarrow{q_{1}}-\overrightarrow{q_{2}}\right|$. The Hamiltonian of our model consists of the Hamiltonian of 


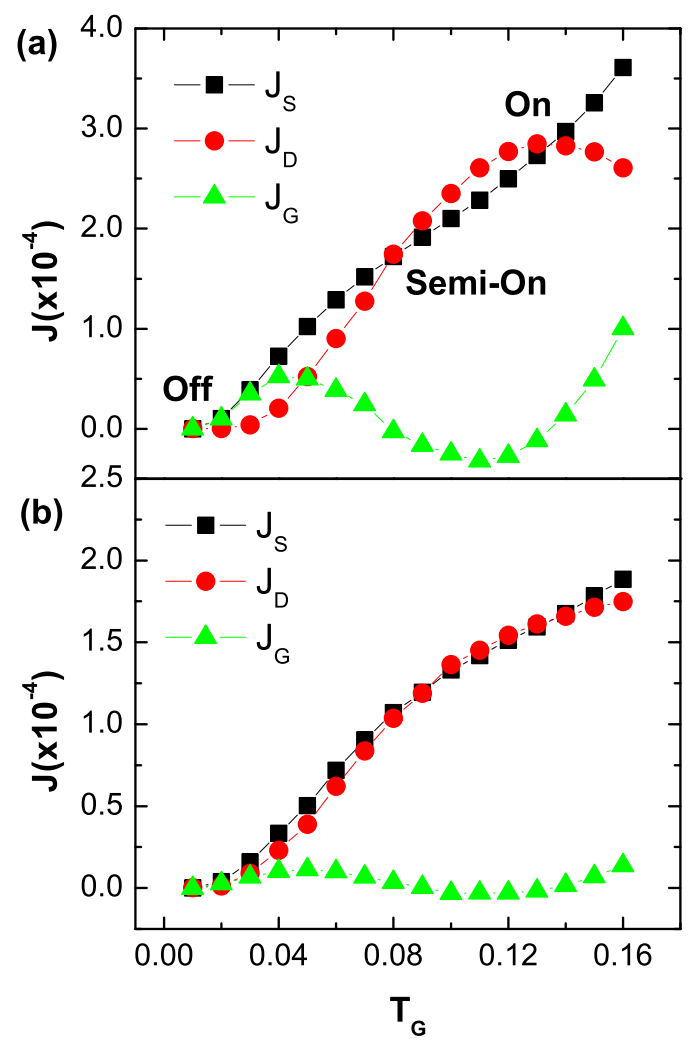

FIG. 2: (a) Thermal switch: a FPU- $\beta$ lattice with $k_{D}\left(=k_{F P U}\right)=0.20$ and $\beta=0.05$ is used as segment $D$. Other parameters remain the same as in Fig. 1 (b). (b) Thermal modulator: all parameters remain the same as in 2 (a) except with $k_{\text {int } S}=0.25, k_{\text {int } D}=0.055$, and $k_{\text {int } G}=0.04$.

each segment:

$$
\begin{array}{r}
H_{W}=\sum_{i=1}^{N_{W, X}} \sum_{j=1}^{N_{Y}}\left[\frac{\left|\vec{p}_{W, i, j}\right|^{2}}{2 m}+V_{W}\left(\Delta r_{W, i, j ; i-1, j}\right)\right. \\
\left.+V_{W}\left(\Delta r_{W, i, j ; i, j-1}\right)-U_{W}\left(\vec{q}_{W, i, j}\right)\right],
\end{array}
$$

and the Hamiltonian of the interface:

$$
\begin{aligned}
H_{\text {int }}= & \sum_{j=1}^{N_{Y}}\left[\frac{\left|\vec{p}_{O, j}\right|^{2}}{2 m}-U_{O}\left(\vec{q}_{O, j}\right)+V_{i n t S}\left(\Delta r_{N_{S, X}, j ; O, j}\right)\right. \\
& \left.+V_{\text {intD }}\left(\Delta r_{N_{D, X}, j ; O, j}\right)+V_{i n t G}\left(\Delta r_{N_{G, X}, j ; O, j}\right)\right] .
\end{aligned}
$$

Similarly, all segments are coupled to each other via harmonic springs. In our model, segments $S$ and $G$ are FK lattices and segment $D$ is FPU- $\beta$ lattice. Thus, $V_{S / G / \text { int } W}(\Delta r)=\frac{k}{2} \Delta r^{2}, \quad V_{D}(\Delta r)=\frac{k}{2} \Delta r^{2}+\frac{\beta}{4} \Delta r^{4}, \quad U_{D}=0$, and $U_{S / G / O}(\vec{q})=\frac{V_{S / G / O}}{(2 \pi)^{2}} \cos 2 \pi q^{x} \cos 2 \pi q^{y}$. The harmonic spring constant $k$ and the on-site potential $V$ may take different values in different segments. Periodic and fixed

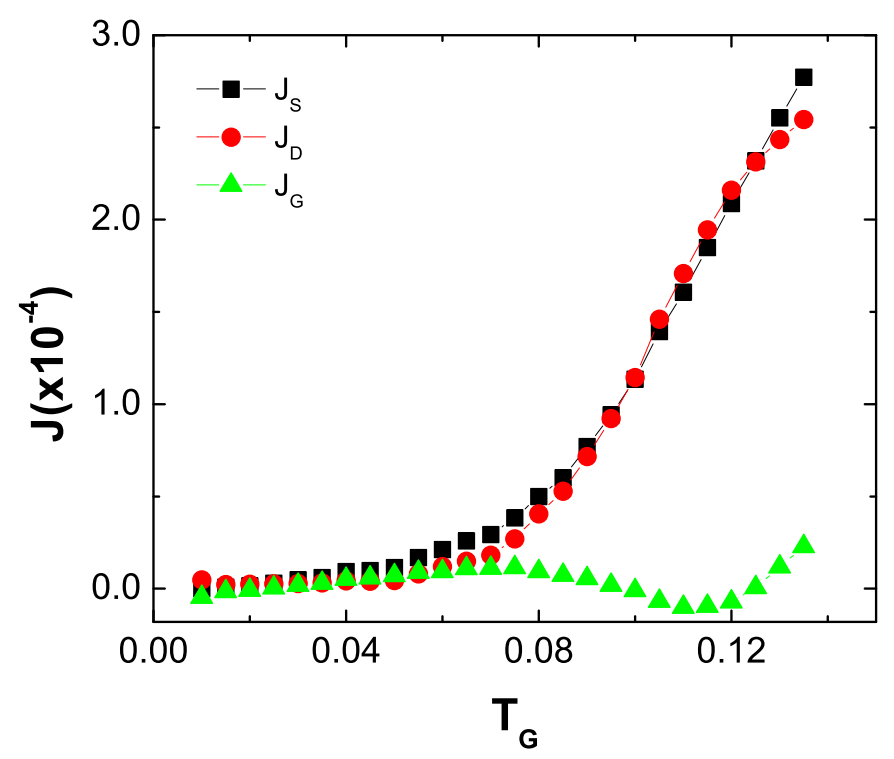

FIG. 3: The thermal modulator in which the FK lattice is used as segment $S$ and the FPU- $\beta$ lattices are used as segment $D$ and $G$. The system parameters are $T_{S}=0.01$, $V_{S}=5.00, \quad k_{S}=0.35, \quad k_{\text {int } S}=0.60 ; \quad T_{D}=0.20, \quad k_{D-F P U}=0.20$, $\beta_{D}=0.05, k_{\text {int } D}=0.05 ; k_{G-F P U}=0.20, \beta_{G}=2.00, k_{\text {int } G}=0.20$, and $V_{O}=5.00$.

boundaries are used in the $\mathrm{Y}$ (transverse) and $\mathrm{X}$ (longitudinal) direction respectively, namely, $\vec{q}_{i_{W}, 0}=\vec{q}_{i_{W}, N_{Y}}$ and $\vec{q}_{0_{W}, j}=(0, j)$ for $j=\left\{1, \ldots, N_{Y}\right\}$. Thus the topologies of the segments, as shown in Fig. 4(a), are cylinders. Nosé-Hoover heat baths are coupled to the ends of segment $(i=1)$. In the stationary state, temperature gradients are set up in the $X$ direction and thus only heat currents through longitudinal links, $J_{i_{W}}\left(=\sum_{j=1}^{N_{Y}} J_{i_{W}, j}\right)$ need to be taken into account, which are constant along each segment.

In Fig. 4(b), we show the heat switching for the 2D thermal transistor model. It can be seen that when $T_{G}$ is at 0.01 and $0.07, J_{S}=J_{D}$ and $J_{G}=0$. At these two points, $J_{D, S}$ are $8.43 \times 10^{-5}$ and $3.38 \times 10^{-4}$ respectively and thus the switching efficiency is about 4 . This is a significant reduction when compared with the $1 \mathrm{D}$ case and this scenario is also quite similar to the $2 \mathrm{D}$ thermal rectifier [5]. We show in Fig. 4(c) that when $T_{S}$ is increased, the difference between $J_{S}$ and $J_{D}$ is reduced, which gives a better modulation of $J_{D, S}$ by the gate temperature. It is worth mentioning that in 2D case, the crossover energy, particles above which are able to overcome the on-site potential barrier is much lower than that in 1D case. Thus the lattice parameters should be re-designed, otherwise the switching efficiency is reduced significantly as shown above. 
(a)
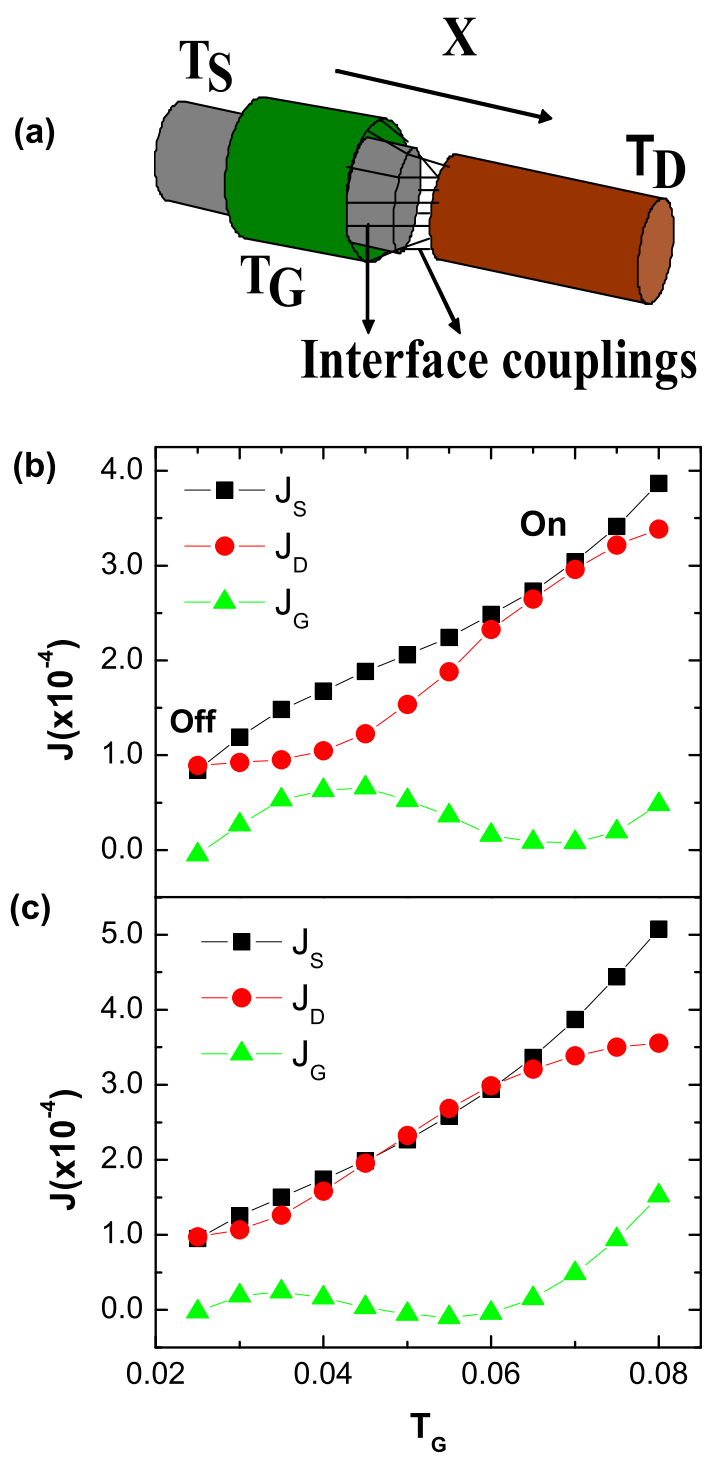

FIG. 4: (a) The prototype of a two-dimensional thermal transistor. Parameters used are (b) Thermal switch: $T_{S}=0.01$, $k_{S}=0.20, \quad V_{S}=5.00, \quad k_{\text {int } S}=0.50 ; \quad T_{D}=0.17, \quad k_{D-F P U}=1.00$, $\beta=3.00, k_{\text {int } D}=0.035 ; k_{G-F P U}=1.00, V_{G}=6.00, k_{\text {int } G}=2.50$, and $V_{O}=5.00$. (c) Thermal modulator with $T_{S}=0.015$. Here, $N_{D, X}=N_{G, X}=10, N_{S, X}=9$, and $N_{Y}=4$.

\section{DISCUSSION AND CONCLUSION}

In summary, we have provided several ways in improving the switching efficiency in $1 \mathrm{D}$ thermal transistor, namely, one can change different lattice in different segments, and/or by adjusting the coupling strength at the interface. Our study has been extended to twodimensional lattices and a prototype of $2 \mathrm{D}$ thermal transistor - a tube-like model have been proposed. Our nu- merical simulations have demonstrated that such a prototype can work as a heat switch and a heat modulator - two basic functions of a thermal transistor.

In our numerical simulations, we have used dimensionless units for the sake of computations. However, all dimensionless units are related to the dimensional physical variables through dimensional scaling and consequently, the physical (real) temperature $T_{r}$ is related to the numerical temperature $T$ through $T_{r}=\frac{m \omega_{0}^{2} a^{2}}{k_{B}} T$ where $k_{B}$ is the Boltzmann constant (please refer to [17] for details). Suppose we use single-walled carbon nanotube as our experimental prototype for the thermal transistor model, namely with the mass of particle $m$, lattice constant $a$, and natural oscillation frequency $\omega_{o}$ correspond to the real values of such a material [19], $T=0.01$ in our simulations corresponds to physical temperature $T_{r}$ of about $100 \mathrm{~K}$. Similar analysis for heat flux gives $J_{r}\left(=m \omega_{o}^{3} a^{2} J\right)$ $\sim 10^{-5} J$. Therefore, with $J \sim 10^{-4}, J_{r}$ is about $10^{-9} \mathrm{~W}$ $(\mathrm{J} / \mathrm{s})$ which is smaller than that one in Ref.[20]. The smaller heat flux obtained in our model is not surprising. It is mainly due to the interacting with external on-site potential [21] and the interface thermal resistance arises in coupling dissimilar nonlinear lattices [4], which is similar to the case of electronic diode where the electrical current are greatly reduced by the P-N junction. For a homogeneous FPU lattice with similar parameters, $J_{r}$ can be about 2 orders of magnitude higher.

Even though a real working prototype for solid state thermal transistor is still an open issue, we believe that the above model or its variants will be realized sooner or later. Moreover, a solid state thermal rectifier using nanotubes has been experimentally demonstrated [22] (see also Ref 23] for other variants). More recently, a tunable and reversible thermal link using multi-walled carbon nanotubes (MWCNTs) 24] were successfully demonstrated whereby the thermal conductance can be controlled mechanically by displacing the outer shell with respect to the inner one. This is a step forward to the realization of thermal transistor. In fact, in the heat control models, be it a thermal rectifier or a thermal transistor, the key factors are: (1) the broken of the spatial symmetry of terminal, namely they are two different materials; (2) nonlinearity in each segment. Therefore, the possible material to be used for the thermal transistor might be multiwalled nanotubes. As the recent experiment 24] shows that the outer wall can be used to control the thermal conductance of the nanotube, thus it can be used as the controlling terminal G.

\section{Acknowledgment}

The work is supported partially by an academic research fund, R-144-000-203-112 from MOE of Singapore, and the Temasek Young Investigator Award of DSTA Singapore under Project Agreement POD0410553 
[1] F. Bonetto, J. L. Lebowitz, and L. Rey-Bellet: Mathematical Physics 2000, edited by A. Fokas, A. Grigoryan, T. Kibble, and B. Zegarlinsky, (Imperial College Press, London, 2000), (pp. 128-150); B. Li, J. Wang, L. Wang, and G. Zhang: Chaos 15 (2005) 015121.

[2] M. Terraneo, M. Peyrard, and G. Casati: Phys. Rev. Lett. 88 (2002) 094302.

[3] B. Li, L. Wang, and G. Casati: Phys. Rev. Lett. 93 (2004) 184301.

[4] B. Li, J. Lan, and L. Wang: Phys. Rev. Lett. 95 (2005) 104302.

[5] J. Lan and B. Li: Phys. Rev. B 74 (2006) 214305.

[6] D. Segal and A. Nitzan: Phys. Rev. Lett. 94 (2005) 034301.

[7] B. Hu, L. Yang, and Y. Zhang: Phys. Rev. Lett. 97 (2006) 124302.

[8] B. Hu and L. Yang: Chaos 15, 015119 (2005).

[9] N. Yang, N. Li, L. Wang, and B. Li: Phys. Rev. B 76 (2007) 020301 (R).

[10] B. Li, L. Wang, and G. Casati: Appl. Phys. Lett. 88 (2006) 143501 .

[11] O.-P. Saira, M. Meschke, F. Giazotto, A. M. Savin, M. Möttönen, J. P. Pekola: Phys. Rev. Lett. 99 (2007) 027203.

[12] L. Wang and B. Li: Phys. Rev. Lett. 99 (2007) 177208.

[13] O. M. Braun and Y. S. Kivshar: Phys. Rep. 306 (1998) 1.

[14] V. L. Pokrovsky and A. L. Talapov: Theory of Incommensurate Crystals, Soviet Scientific Reviews Supplement Series, Physics Vol. 1 (Harwood, New York, 1984).

[15] S. Nosé: J. Chem. Phys. 81 (1984) 511; W. G. Hoover,
Phys. Rev. A 31 (1985) 1695.

[16] K. R. Patton and M. R. Geller: Phys. Rev. B 64 (2001) 155320.

[17] B. Hu, B. Li, and H. Zhao: Phys. Rev. E 57 (1998) 2992 .

[18] K. Aoki and D. Kusnezov: Phys. Rev. Lett. 86 (2001) 4029; H. Kaburaki and M. Machida, Phys. Letts. A 181 (1993) 85.

[19] For carbon atom, with the mass $m=1.98 \times 10^{-26} \mathrm{~kg}$, lattice constant $a \sim 10^{-10} \mathrm{~m}$, and the natural oscillation frequency $\omega_{o} \sim 4.53 \times 10^{13} \mathrm{~s}^{-1}$ (if we take for example $\mathrm{E}=30 \mathrm{meV}$ [25]), $T_{r} \sim 2.95 \times 10^{4} T$ and $J_{r} \sim 1.84 \times 10^{-5} J$.

[20] G. Zhang and B. Li: J. Chem. Phys. 123 (2005) 114714. For a $(9,0)$ single-walled carbon nanotube whose ends are thermalized with Nosé-Hoover heat baths at $T_{r}=310 \mathrm{~K}$ and $290 \mathrm{~K}, J_{r}$ is about $5.26 \times 10^{-7} \mathrm{~W}$. With roughly 4 carbon atoms per cross section of the nanotube, $J_{r}$ can be further approximated to about $10^{-7} \mathrm{~W}$.

[21] D. Donadio and G. Galli: Phys. Rev. Lett. 99 (2007) 255502.

[22] C. W. Chang, D. Okawa, A. Majumdar, and A. Zettl: Science 314 (2006) 1121.

[23] G. Wu and B. Li: Phys. Rev. B 76 (2007) 085424.

[24] C. W. Chang, D. Okawa, H. Garcia, T. D. Yuzvinsky, A. Majumdar, and A. Zettl: Appl. Phys. Lett. 90 (2007) 193114.

[25] S. Rols, Z. Benes, E. Anglaret, J. L. Sauvajol, P. Papanek, J. E. Fischer, G. Coddens, H. Schober, and A. J. Dianoux: Phys. Rev. Lett. 85 (2000) 5222. 